DENTAL PUBLIC HEALTH; CARDIOLOGY

\section{Loss of teeth and coronary heart disease}

Ragnarsson E, Eliasson ST et al. Int J Prosthodont 2004; 17: 441-446

Tooth loss and heart disease were linked only by their common causal factor of tobacco smoking.

There is current interest in whether periodontal disease may cause other illnesses, such as heart disease. Early studies suggested such a link, but evidence has been accumulating to suggest that smoking is a major confounding factor. The present study involved two samples of Icelandic subjects: 1,023 from an Icelandic Heart Society study, and 1,590 from a WHO cardiovascular disease project.

During the studies, which lasted respectively from 1966 to 1998, and 1987 to 1998, 353 subjects died, 82 of them from heart disease. From all CHD mortality data, adjusted odds ratios were calculated, allowing for known risk factors in the order: age and gender, cholesterol, blood pressure, educational level and smoking. When edentulousness was considered, significant but very small hazard ratios were obtained (means 1.88 to 1.46) until smoking was added (1.46; CIs 0.88-2.43); number of yrs of edentulism and number of remaining teeth gave totally insignificant hazard ratios throughout.

doi:10.1038/sj.bdj.4812177

\section{PERIODONTOLOGY; CARDIOLOGY}

\section{Periodontal pathogens in atheromatous}

plaques. A controlled clinical and laboratory trial

Cairo F, Gaeta C et al. J Periodont Res 2004; 39: 442-446

No periodontal pathogens were found in atheromatous plaques.

Some studies have suggested an association where periodontitis is a possible cause of cardiovascular disease, but others have indicated that smoking is a common risk factor for both, and have even questioned whether there is any association when all factors are considered. In this study, a test group of 26 dentate patients who received carotid endarterectomy was examined periodontally, including dental plaque sampling, and their carotid atheromatous plaques were compared with those removed from a control group of 26 edentulous patients undergoing the same procedure.

In the test group, 7 patients were excluded as negative to DNA amplification, and in the control group, 5. Using a PCR method with tested specific primers, $T$. forthsythensis was found in dental plaque from $79 \%$ of test group patients, $F$. nucleatum in 63\%, $P$. intermedia in 53\%, P. gingivalis in 37\%, and $A$. actinomycetemcomitans in 5\%. Carotid specimens of all 40 patients showed severe atherosclerosis, but none of the periodontal bacteria were detected. Tests showed the experimental method to be extremely sensitive, with a detection limit of 100 genome equivalents.
IMPLANT DENTISTRY

\section{Comparison of implant survival following sinus floor augmentation procedures with implants placed in pristine posterior maxillary bone: a systematic review} Graziani F, Donos N et al. Clin Oral Imp/ Res 2004; 15: 677-682

Implant survival in different studies showed greater variability in grafted areas than those without augmentation.

Implant survival in the posterior maxilla is less than in other parts of the jaws. This study sought to compare implant survival after sinus grafting with survival after normal placing of implants in this region. After initial identification of 579 studies possibly relevant to this comparison, 486 were rejected as clearly irrelevant, and a further 87 were excluded because of lack of control or comparison groups.

In the remaining 6 studies, individual implant survival ranged from $75 \%$ to $100 \%$ in both the augmented and non-augmented posterior maxilla, with follow-up from 8 months to 6 yrs. Patientbased implant survival could only be estimated from 2 studies, at 36\% after 3 yrs with iliac block bone graft, and 100\% after 5 yrs with bone from intra-oral sources. Respective controls gave survival of 73\% and 100\%. The authors were unable to perform a meta-analysis because of the heterogeneity of studies.

doi:10.1038/sj.bdj.481279

\section{PROSTHODONTICS; BEHAVIOURAL SCIENCE}

\section{The dentist's communicative role in} \section{prosthodontic treatment}

\section{Sondell K, Palmqvist S et al. Int J Prosthodont 2004; 17: 666-671}

Dentist communication influenced the patient's opinion at the time, but had no apparent effect on the patient's view of the completed treatment.

There is evidence that dentists need to communicate well to achieve predictable treatment outcomes. However, patients have been studied more than dentists, and this study aimed at identifying the dentist's communicative role in patient satisfaction with prosthodontic treatment. In 3 Swedish specialist prosthodontic clinics, 61 patients treated by 15 dentists were followed through treatment with conversation recorded, and completed appropriate questionnaires.

Following statistical multilevel modelling, patient satisfaction during an appointment was found to be largely related to patient factors, with only $2 \%$ of variance dependent on the dentist in a communication model. Three months after completion of treatment, patient satisfaction did not appear to relate to the dentist. The authors comment that dentist communication affected patient satisfaction during an appointment, but had no impact on patient satisfaction with outcome shortly after treatment.

doi:10.1038/sj.bdj.4812178 